\title{
Criminologie
}

\section{Significations et interprétations de la gravité des événements violents : le cas des voies de fait traitées à la Cour municipale de Montréal}

\section{Éva Nonn}

Volume 24, numéro 2, 1991

Regard sur les tribunaux

URI : https://id.erudit.org/iderudit/017308ar

DOI : https://doi.org/10.7202/017308ar

Aller au sommaire du numéro

Éditeur(s)

Les Presses de l'Université de Montréal

ISSN

0316-0041 (imprimé)

1492-1367 (numérique)

Découvrir la revue

Citer cet article

Nonn, É. (1991). Significations et interprétations de la gravité des événements violents : le cas des voies de fait traitées à la Cour municipale de Montréal. Criminologie, 24(2), 31-55. https://doi.org/10.7202/017308ar
Résumé de l'article

Based on the results of an empirical study on the judicial treatment of cases of assault and battery at the Municipal Court of Montreal, this article focuses on one particular aspect of this treatment, the classification of certain acts as infractions. Its object is to describe and analyze the socio-political and juridic factors that can influence the decisions made by the police and prosecutors when pronouncing the charges. By comparing these charges with the legal and extra-legal variables, we see a difference in the classification of similar cases depending on the family or social context. This supposes some difficulty in defining the criminal gravity of acts committed in conflicts involving parties bound by other bonds as well as their case before the court. 


\title{
SIGNIFICATIONS ET INTERPRÉTATIONS DE LA GRAVITÉ DES
} ÉVÉNEMENTS VIOLENTS : LE CAS DES VOIES DE FAIT TRAITÉES À LA COUR MUNICIPALE DE MONTRÉAL Éva Nonn ${ }^{1}$

\begin{abstract}
Based on the results of an empirical study on the judicial treatment of cases of assault and battery at the Municipal Court of Montreal, this article focuses on one particular aspect of this treatment, the classification of certain acts as infractions. Its object is to describe and analyze the socio-political and juridic factors that can influence the decisions made by the police and prosecutors when pronouncing the charges. By comparing these charges with the legal and extralegal variables, we see a difference in the classification of similar cases depending on the family or social context. This supposes some difficulty in defining the criminal gravity of acts committed in conflicts involving parties bound by other bonds as well as their case before the court.
\end{abstract}

Une récente étude que nous avons faite sur le traitement des cas de voies de fait à la Cour municipale de Montréal ${ }^{2}$ est à l'origine de cet article. Les données de cette recherche empirique concernent 625 accusés sur un total de 2525 cas ayant fait l'objet d'une telle accusation à la Cour municipale de Montréal, au cours de la période du 30 septembre 1986 au $1^{\text {er }}$ octobre 1987. Elles ont été recueillies à partir des différents documents judiciaires (procèsverbaux, feuilles de conciliation) et policiers (rapports initiaux, précis de fait, demandes d'intenter des procédures). Les procureurs de la Couronne, dans les interviews qu'ils nous ont accordés, nous ont aidée à comprendre à la fois le fonctionnement de la procédure de conciliation qu'ils ont mise en place ${ }^{3}$ et la nature des difficultés auxquelles ils font face lorsqu'ils procèdent dans des cas intrafamiliaux où la solution adéquate des conflits dépasse souvent leurs compétences.

1. Agente de recherche au Groupe de recherche informatique et droit (GRID), Université du Québec à Montréal, C.P. 8888, Succ. A, Montréal (Québec) H3C 3P8.

2. É. Nonn (1990), «Facteurs significatifs dans le traitement des cas de voies de fait à la Cour municipale de Montréal», rapport de recherche du Groupe de recherche et d'analyse sur les politiques et pratiques pénales, sous la direction de P. Landreville, professeur, École de criminologie. en 1985 .

3. Projet pilote mis sur pied par les procureurs de la Couronne de la Cour municipale 
Nous sommes consciente des limites méthodologiques de nos résultats découlant du fait que nous ayons traité des données empiriques fournies par les organismes pénaux à partir d'une grille de sélection particulière sur leur propre activité et sur les caractéristiques de la population qu'ils ont à traiter. Il est évident que les messages véhiculés reflètent inévitablement les objectifs et les idéologies des émetteurs. En dépit de ces limites, nous espérons cependant que nos données empiriques révèlent d'autres éléments du réel que la reconstruction de celui-ci, comme cela aurait pu être le cas si nous avions choisi d'analyser cette réalité juridico-pénale au moyen des interviews effectués avec les acteurs judiciaires ou avec les parties impliquées dans les conflits traités par les organismes pénaux.

Par ailleurs, nos données empiriques ne peuvent témoigner du vécu des victimes avant ou après les processus pénaux entamés, ni de leur perception de la gravité de ces actes qui peuvent être qualifiés de voies de fait. Elles peuvent néanmoins faire état de leur attitude face au système pénal. En effet, nos données permettent de croire que les décisions des acteurs pénaux sur les accusations à porter sont influencées par la signification que semblent donner aux actes les parties impliquées à travers leur attitude face aux instances pénales.

Inspirée par les changements survenus au cours des années 1980 tant sur le plan de la politique pénale que de celui du contrôle social de la violence conjugale $^{4}$, notre recherche avait pour objectif de décrire et d'analyser les incidences socio-politiques et juridiques susceptibles d'intervenir dans le processus de décision des agents ou des parties impliqués dans les conflits lors des procédures pénales.

À partir de certains résultats de cette recherche et des écrits disponibles sur le sujet, nous consacrons cet article à la qualification des actes, c'est-à-dire à un élément important du processus de transformation de certains événements violents en infractions de voies de fait. La qualification juridico-pénale des actes s'applique à toutes les phases pénales, depuis la plainte déposée à la police, la dénonciation et la déclaration de la poursuite jusqu'à la décision finale des juges. C'est à partir de cette activité que la clientèle est sélectionnée et dirigée vers les différents paliers judiciaires (une cour inférieure ou supérieure). La qualification des actes est par ailleurs en rapport avec l'imposition de mesures privatives de liberté (arrestation, détention sous garde ou détention provisoire) tout autant qu'avec la sélection des cas pour la procédure de conciliation (pouvant permettre à certains accusés d'éviter les procès). Finalement,

4. Voir parmi ces changements l'adoption en 1986 d'une nouvelle politique d'intervention québécoise en matière de violence conjugale par les ministères de la Justice et du Solliciteur général. 
elle influe sur le verdict et sur la nature et le quantum de la sentence qui sera imposée.

L'analyse du processus de qualification des actes permet aussi de se pencher sur la question fondamentale de leur gravité. Par ailleurs, les écrits spécialisés indiquent que l'appréciation de la gravité des événements intervient à la fois dans les décisions des parties impliquées et dans celles des acteurs du système pénal. Il nous a semblé pertinent de chercher à savoir si les évaluations de la gravité des événements aux différents niveaux concordaient ou pouvaient concorder.

\section{L'INTERPRÉTATION DE LA GRAVITÉ DE L'ACTE COMME ÉLÉMENT DE PRISE DE DÉCISIONS JUDICIAIRES : CRITIQUES ET PROPOSITIONS}

\section{LA SÉLECTIVITÉ DES DÉCISIONS JUDICIAIRES}

La qualification des actes, on l'a vu, est la pierre angulaire du mécanisme de décisions des acteurs du système pénal. D'importantes recherches sur ce sujet ont étudié la sélectivité, l'arbitraire ou la disparité de ces mécanismes. Théoriques ou empiriques, ces recherches se sont généralement proposé d'enquêter sur une instance particulière du système pénal ou un type particulier de décision. C'est ainsi qu'ont été analysées les décisions des agents de services policiers (Ericson, 1981; Ericson et Baranek, 1982), des avocats de la Couronne ou de la défense (Poirier, 1988) ou des juges de différentes cours (Sellin, 1928; Hogarth, 1971; Myers, 1987; Black, 1989). Quant aux études sur un type particulier de décisions, elles se sont penchées surtout sur les facteurs qui interviennent dans les décisions concernant les mesures privatives de liberté telles l'arrestation ou la détention (Freedland, 1963; Nagel, 1983; Cousineau, Laberge et Théoret, 1986), la libération conditionnelle (BenzvyMiller, 1988; Brodeur, 1990) ou la détermination de la peine.

À la lumière de l'importance accordée à la gravité de l'acte lors de la détermination de la peine, nous reprendrons quelques apports théoriques sur les facteurs qui interviennent dans les décisions au cours de cette phase judiciaire. Avant de considérer ces facteurs, il convient toutefois de souligner que la détermination de la peine n'est en fait que l'aboutissement des «processus formels et informels» complexes qui la précèdent et la marquent (Robert et Faugeron, 1981; Landreville et al., 1981; Robert, 1985). En effet, tous les comportements violents ne sont pas définis comme des infractions pénales, pas plus que ce ne sont les comportements qu'on a déclarés criminels, et ces derniers ne sont pas tous pris en charge par les organismes pénaux. À titre d'exemple, on pourrait citer la Commission de réforme du droit du Canada qui, en traitant de la normalisation des cas de voies de fait, affirme 
que dans notre société démocratique, les comportements violents devraient tous être sanctionnés également. Cependant, compte tenu de la «demande différentielle» quant à l'intervention étatique à l'égard des comportements violents de différentes natures, la commission propose de ne pas considérer les gestes violents posés dans les différents domaines du sport, alors qu'elle favorise une intervention pénale plus stricte à l'endroit de la violence conjugale. Au-delà de cette distinction entre les différents types de violence physique, nous pourrions nous interroger également sur le caractère social de cette manière de définir la violence. En définissant la violence en termes de violence physique, on tend à viser certaines couches sociales plus que d'autres, alors qu'on pourrait envisager la violence selon une interprétation plus large qui inclurait, par exemple, les incidents dans les domaines de la politique ou du travail, ce qui toucherait également les couches sociales mieux nanties.

Les auteurs soulignent également que les moyens sociaux mis en œuvre pour régler les conflits criminalisés sans intervention pénale ne sont pas distribués équitablement. Selon le contexte social des événements et les parties impliquées, des comportements semblables du point de vue «juridico-pénal» n'ont pas le même degré de visibilité (Chapman, 1968) ni les mêmes chances d'être vus par la police, d'être «rapportés» par la victime ou par les intermédiaires (Goffman, 1968; Robert et Faugeron, 1981). Quant aux parties mêmes, celles qui ont à leur disposition d'autres services (financiers, médicaux ou sociaux) éviteront l'acheminement vers le pénal (Landreville, 1983).

Dans les cas où le conflit est interprété et accepté comme une infraction pénale, d'autres facteurs s'ajoutent pour qu'il reçoive effectivement une solution pénale. Les différentes politiques criminelles en cours, les conceptions fonctionnelles et idéologiques des agents influencent la survie de la cause et la ventilation des affaires vers les «étages» subséquents (Robert et Faugeron, 1981). En ce sens, les décisions politiques sur la nature de l'intervention pénale face à la violence conjugale peuvent affecter l'activité des policiers lorsqu'ils interviennent dans les conflits intrafamiliaux en optant pour le renvoi de la cause à la Cour plutôt que pour la fermeture du dossier.

Quant aux études critiques concernant les décisions judiciaires sur les sentences (Sellin, 1928; Hagan, 1974; Hogarth, 1971 et 1975; Myers, 1971 et 1987), elles démontrent souvent un traitement différentiel des cas dû à l'écart entre les principes de la justice et la pratique pénale. À l'origine de ce traitement différentiel, on retrouve une pratique judiciaire où l'application de la loi devient secondaire par rapport aux considérations extra-légales, que celles-ci soient relatives à la perception des agents ou à certaines caractéristiques des parties, des causes, du fonctionnement des organismes et des idéologies. 
Ainsi, Black (1989) a montré le rôle marquant de la dynamique sociale dans le traitement judiciaire des cas lorsqu'il a analysé la complexité et l'interdépendance des variables statutaires des causes, des parties et des agents judiciaires engagés dans les décisions. L'auteur souligne que les accusés ne subissent pas le même traitement selon leur provenance sociale et leur stabilité d'emploi; d'autre part, la situation socio-économique des victimes peut également influer sur les décisions des agents qui ne s'impliquent pas de la même façon selon le statut social de la victime. Le statut social des juges, la distance sociale qui les sépare ou les rapproche des parties et leur place dans la hiérarchie judiciaire peuvent influencer leur perception de la gravité de l'acte et la nature de la sentence imposée. Le statut social des avocats, leur expérience, le type de services qu'ils offrent (pratique privée ou aide juridique) peuvent aussi jouer. Finalement, le caractère social des causes intervient aussi dans cette dynamique, puisque les cas qui rapportent des bénéfices (financiers ou professionnels), qui font la manchette des médias ou qui sont considérés comme faciles à juger à cause du contexte social suscitent un plus gros investissement de la part des agents.

Feeley (1979) affirme à son tour qu'outre le statut social des parties et des tiers, celui de l'institution judiciaire peut affecter les décisions. Ainsi, les cours criminelles dont la juridiction se limite aux causes de "gravité mineure» (ce pourrait être le cas de la Cour municipale) traitent généralement les conflits qui relèvent surtout de problèmes d'ordre social (pauvreté, chômage, alcoolisme, toxicomanie, troubles psychologiques) et où la solution exigerait des moyens dont ne dispose pas le système judiciaire. Cette situation risque de démotiver les acteurs de ces cours, la solution des conflits exigeant d'eux un travail d'intervenant social alors que leurs collègues des cours supérieures font $d u$ «droit».

\section{LE PRINCIPE DE PROPORTIONNALITÉ: UNE RÉPONSE POSSIBLE À LA DISPARITÉ DES SENTENCES}

Deux types de réponses nous semblent pouvoir être apportés à ce problème de la sélectivité, de l'arbitraire et de la disparité des décisions judiciaires. Préconisant l'éloignement du «culte de la loi», certains proposent d'étudier les normes pénales elles-mêmes (Pires et Landreville, 1985), l'incidence des normes sociales sur celles-ci, l'impact de l'activité ou de la passivité de certains groupes sociaux ou d'entrepreneurs moraux sur la criminalisation des comportements (Becker, 1963; Landreville, 1983) ainsi que l'effet de ces normes dans la gestion différentielle de la population carcérale (Cousineau, Laberge et Théret, 1986). 
D'autres auteurs, qui ne remettent en cause ni la légitimité des normes pénales, ni la nature ou les priorités des politiques les concernant, visent plutôt un meilleur ajustement, un meilleur accord entre politiques et pratiques pénales, l'efficacité des unes devant passer par la structuration des autres. S'inscrivant dans cette perspective, les travaux proposant des modèles aux fins d'unification de la pratique sentencielle aboutissent souvent à définir des lignes directrices en vue de guider ou de structurer la prise de décision des juges. Divergentes quant aux restrictions qu'elles imposent aux juges, ces lignes directrices ont en commun de $s$ 'inspirer davantage des principes du just desert ou de la juste rétribution que de ceux de la réhabilitation du contrevenant.

C'est sous ce jour qu'il faut voir les travaux de la Commission canadienne sur la détermination de la peine (1987) qui, en reprenant les résultats d'analyses démontrant le peu de succès qu'ont connu les objectifs de réhabilitation, de dissuasion, de neutralisation ou de dénonciation, propose de «promouvoir le respect» de la loi «par l'imposition de sanctions justes», ce qui permettra de faire voir aux citoyens, du fait de la prévisibilité des sanctions, «qu'ils seront tenus de rendre compte de leur comportement illicite, et que le prix qu'ils auront à payer sera plus élevé que le bénéfice qu'ils pourraient en espérer». C'est dans cette logique qu'il faut considérer comme un "principe prépondérant ", lors de la détermination de la peine, sa proportionnalité à la gravité de l'acte et au degré de responsabilité de son auteur ${ }^{5}$.

La prépondérance accordée au principe de proportionnalité a suscité un vif débat qui dure encore (Brodeur, 1989 et 1990; Gabor, 1990; Pires, 1987 et 1990; Von Hirsch, 1990). Ainsi, le rapport du Comité permanent de la justice et du Solliciteur général présidé par D. Daubney (1988), rapport intitulé «Les responsabilités à assumer», insiste à la fois sur la responsabilité, la réadaptation du délinquant, la dénonciation et la neutralisation ainsi que sur la réparation du tort fait à la victime. Il semble toutefois que l'importance accordée à la proportionnalité des peines en rapport avec la gravité de l'acte commis et avec le degré de culpabilité de l'accusé demeure un point commun dans un grand nombre de discours canadiens, tant officiels que théoriques (Ruby, 1986 et 1987; Cusson, 1988; Brodeur, 1990).

$S$ 'il y a accord sur la pertinence du principe de proportionnalité, il semble cependant qu'il y ait mésentente quant aux facteurs devant ou pouvant être considérés lors de la détermination de la gravité «objective» et «relative» de l'acte et les sanctions proportionnelles à celle-ci (Ruby, 1987; Robert, 1988). Il convient de noter qu'en parlant de la gravité «objective» de l'acte, on fait référence aux dispositions du Code criminel et aux peines maximales

5. CCDP, p. 166. 
qui y sont définies. Critiquées pour leur nature sélective (Pires et Landreville, 1985), pour leur ambiguïté et leur désuétude (CRDC, 1986) ainsi que pour les peines trop sévères et rarement appliquées qu'elles proposent (CCDP, 1987), ces dispositions ne permettent apparemment pas aux acteurs judiciaires de porter un choix éclairé concernant la gravité objective de l'acte et de la peine équitable dans un cas concret. Par gravité «relative» (ou «subjective»), on entend généralement celle des circonstances particulières de l'incident et les différentes circonstances aggravantes ou atténuantes. Si les juges soulignent la complexité de ces circonstances, certains auteurs, tels Doob et Park (1987), soutiennent que dans les faits, elles sont rarement prises en considération. Ainsi, dans des cas similaires, ce ne seraient pas les mêmes circonstances qui seraient évoquées (Benzvy-Miller, 1988, Ruby, 1987).

\section{FACTEURS POUVANT INFLUENCER LES PROCESSUS DE QUALIFICATION DES INCIDENTS VIOLENTS}

Les facteurs pouvant intervenir dans la qualification des actes violents peuvent être regroupés en fonction des catégories suivantes:

- les facteurs liés aux mouvements sociaux et aux décisions politiques en matière de violence;

- les définitions légales que donnent des voies de fait le Code criminel et les dispositions administratives;

- les facteurs liés au statut social des parties impliquées et au caractère social des événements;

- les variables liées à l'administration de la justice, aux décisions prises antérieurement.

Après une brève discussion des facteurs liés aux deux premières catégories, nous présenterons l'analyse des données que nous avons recueillies à la Cour municipale et à la police. Dans un premier temps, nous traiterons des données concernant les caractéristiques socio-économiques et juridiques des parties impliquées et des facteurs liés aux événements en cause. Dans un deuxième temps, nous verrons l'impact que ces facteurs peuvent avoir sur la qualification des actes.

\section{LES MOUVEMENTS SOCIAUX ET LES DÉCISIONS POLITIQUES COMME ÉLÉMENTS SUSCEPTIBLES D'INTERVENIR DANS L'INTERPRÉTATION DES ÉVÉNEMENTS VIOLENTS}

Parmi les changements socio-politiques pouvant affecter le traitement pénal des cas de voies de fait, mentionnons d'abord certains groupes sociaux 
qui réclament une plus grande intervention étatique dans le domaine de la sécurité publique tout en souhaitant une simplification de son administration de façon à en réduire les coûts. Comme le Comité d'étude sur les solutions de rechange à l'incarcération (1986) l'a souligné, ces changements d'orientation risquent toutefois d'avoir des conséquences négatives. La diminution du budget de l'État peut impliquer notamment une diminution des services sociaux et médicaux et, par conséquent, une prise en charge de certains problèmes sociaux par les organismes pénaux plutôt que par ces services. Les personnes affligées de problèmes d'ordre psychologique, par exemple, risquent d'être prises en charge par les organismes pénaux.

Par ailleurs, nous pourrions mentionner l'impact considérable du mouvement féministe et son implication dans le contrôle social de la violence conjugale. À ce propos, N. Davis (1988) rapporte que dans les années 1980, la déception qu'a suscitée l'inefficacité des interventions des services d'aide (étatiques ou communautaires) a mené à une alliance entre le mouvement féministe et l'État pour criminaliser la violence conjugale, ce qui a entraîné une série de mesures coercitives telles qu'une augmentation des arrestations et une politique sentencielle plus ferme. Si Davis estime que la violence conjugale a diminué à la suite de ces mesures, elle déplore cependant l'extension du contrôle étatique sur la vie familiale et, surtout, le contrôle plus serré des groupes sociaux défavorisés ${ }^{6}$. Remarquons que la politique d'intervention en matière de violence conjugale au Québec (1986) semble refléter cette liaison entre politiques pénales et mouvement féministe des années 1980. Favorisant l'intervention pénale et insistant sur la dénonciation des comportements violents, cette politique tranche le débat sur la nature du contrôle social de la violence conjugale en réintégrant la résolution de ces conflits dans le système pénal ${ }^{7}$.

Si nos données ne permettent pas de mesurer l'impact de ces changements sociaux et politiques sur l'appréciation pénale de la gravité de tels incidents violents, les statistiques criminelles sur la violence conjugale compilées par les services de police de la province du Québec nous montrent clairement une prise en charge croissante de ce type d'événements dans un contexte où le nombre des événements en tant que tels est peut-être demeuré inchangé. En effet, ces statistiques signalent qu'en 1987, sur 40284 crimes violents, 6559 cas de violence conjugale ( $16 \%$ ) ont été rapportés, dont 63,7 pour cent (4 177) pour voies de fait et 17 pour cent pour menaces. En 1988, le nombre d'infractions reliées à la violence conjugale passe à 8096 , ce qui représente

6. N. Davis (1988), p. $360-365$.

7. Sur les options théoriques et empiriques, voir l'analyse de H. Dumont (1986). 
une hausse de 23,4 pour cent. Parmi ces cas, 78,4 pour cent sont des voies de fait $^{8}$.

\section{L'INTERPRÉTATION LÉGALE DE LA GRAVITÉ DES VOIES DE FAIT}

Pour déterminer la gravité de l'acte, les juges devraient, du moins en principe, se baser d'abord sur les dispositions du Code criminel. Il semble toutefois que ces dispositions ne facilitent pas la qualification des voies de fait. D'abord, les articles concernant cette infraction sont disséminés dans différentes parties du Code, et ce de façon anarchique. Non seulement les définitions légales sont-elles mal agencées dans le Code, mais elles sont formulées d'une façon ambiguë, ce qui rend difficile leur interprétation judiciaire. Par exemple, elles n'expliquent pas clairement ce que l'on doit entendre par «lésions corporelles». Dans une disposition qui les définit comme des «blessures qui nuisent à la santé ou au bien-être du plaignant et qui ne sont pas de nature passagère ou sans importance», le Code criminel ne permet pas de distinguer sans équivoque les gestes violents selon leur gravité, les termes «nature passagère» et «sans importance» étant trop vagues dans le cas de blessures physiques et plutôt inopérants dans le cas de blessures psychiques.

Abstraction faite des articles concernant l'homicide, ce sont les articles 265 et 266 du Code criminel $^{9}$ qui contiennent les principales dispositions en matière de violence. En outre, on retrouve plusieurs articles disséminés dans d'autres parties du Code criminel qui régissent les mêmes comportements violents ou des comportements analogues, certains ayant trait aux circonstances particulières entourant les événements, d'autres venant aggraver la cause de par la nature particulière de la victime (voies de faits contre les agents de la paix). Enfin, certaines infractions, en raison des moyens utilisés ou de la nature des blessures infligées, peuvent aussi encourir des sanctions plus graves (agression armée, lésions corporelles).

L'ambiguité de la définition de la gravité de l'acte dans les cas d'infractions «mixtes» vient s'ajouter à la dispersion des infractions semblables dans plusieurs parties du Code criminel, ce qui ne facilite pas la qualification des actes d'une manière uniforme. Ces infractions pouvant être poursuivies en tant qu'actes criminels ou en tant qu'infractions punissables par voie de déclaration sommaire de culpabilité, c'est aux procureurs de la Couronne que

8. Statistiques sur la violence conjugale, 1987-1988.

9. Notons que nous utilisons dans cet article la nouvelle numérotation des articles du Code criminel, alors qu'au moment où des accusations ont été portées l'ancienne était en vigueur. Ce changement n'a pas affecté le contenu de ces normes; c'est pourquoi nous ne trouvons pas utile de renvoyer à des articles anciens. 
revient le choix de porter des accusations d'avoir commis l'un ou l'autre type d'infraction. Les conséquences de ce choix sont importantes. Une peine maximale de cinq ans d'emprisonnement dans le premier cas, une peine de six mois dans le deuxième. De plus, en cas d'acte criminel, l'accusé doit comparaître en personne, et à défaut de comparaître, un mandat d'arrestation est émis contre lui. Par contre, si le prévenu est accusé d'une infraction punissable par voie de déclaration sommaire de culpabilité, l'émission d'un mandat sera optionnelle lorsqu'il est représenté par un avocat. L'obligation pour les prévenus accusés d'un acte criminel de subir le prélèvement d'empreintes digitales est une autre conséquence de ce choix. Ces accusés peuvent également faire l'objet d'une enquête préliminaire. Mentionnons enfin le droit de la personne accusée d'un acte criminel de choisir le mode de procès. Malgré les conséquences majeures pouvant en découler, les législateurs n'ont élaboré aucun critère relatif à ce choix. Il revient aux procureurs de décider des circonstances aggravantes qui influenceront la décision. Remarquons que les agents policiers jouent aussi un rôle important à cet égard, puisqu'ils sont les premiers à qualifier les actes.

\section{LES CARACTÉRISTIQUES SOCIO-ÉCONOMIQUES DES PARTIES IMPLIQUÉES ET LES ÉVÉNEMENTS}

Comme nous l'avons déjà souligné, une des caractéristiques du traitement judiciaire des cas par les cours inférieures est le fait qu'elles traitent largement des infractions de gravité mineure commises par une population de condition socio-économique très précaire. Si cela est susceptible d'influer sur la façon de résoudre les causes de voies de fait de façon générale, ce l'est encore davantage dans les cas d'incidents intrafamiliaux où la relation entre les parties impliquées ne peut être restreinte uniquement à celle de «victime-agresseur». Avec ou sans rupture des liens, cette relation, dans bien des cas, se maintiendra après l'intervention pénale. La grille de lecture pour chaque cas n'est pas nécessairement la même pour les parties impliquées, le système pénal ou même pour d'autres personnes dans une situation similaire au plan «juridicopénal». À cet égard, deux questions peuvent se poser: les victimes veulentelles que leur conflit, semblable selon les définitions légales, soit traité d'une façon semblable? Est-il possible de le traiter de façon semblable?

Quant à la première question, les études victimologiques ${ }^{10}$ démontrent que les attentes des victimes face à la justice ne sont que rarement satisfaites. Les interviews que nous avons faites avec des procureurs de la Cour municipale confirment cette insatisfaction des conjointes victimes. Dans les cas où l'acte violent n'est que le dernier incident (peut-être même pas le plus

10. Baril (1984). 
grave) d'un long processus, les victimes s'attendent à ce que les juges prennent des mesures sévères contre leur conjoint, alors qu'il ne sera peut-être pas sanctionné sévèrement faute d'antécédents judiciaires ou parce que les incidents familiaux précédents n'ont pas êté rapportés. Dans d'autres cas, l'accusé, soit parce qu'il a un casier judiciaire, soit parce que l'incident a causé des blessures graves, sera emprisonné alors que la victime ne désire pas l'incarcération de son conjoint avec qui, malgré les événements, elle souhaite continuer à vivre.

Quant à la deuxième question, D. S. Elliott (1989), reprenant des analyses effectuées à ce sujet, mentionne la difficulté pour les intervenants judiciaires de mener au procès les cas intrafamiliaux où souvent la victime est le seul témoin des événements. Dans les rares cas où des tiers sont présents lors de l'incident, ce sont des parents des parties qui, dans un cas sur deux, refusent de collaborer avec la poursuite. L'auteur rapporte par exemple la pratique courante de la poursuite aux États-Unis qui, pour éviter ce genre de problèmes, ne s'engage pas s'il n'y a pas au moins deux témoins présents ${ }^{11}$.

Les interviews réalisées avec les procureurs de la Couronne témoignent également de la difficulté qu'ils éprouvent à concilier la volonté manifestée par la victime avec son intérêt et avec «l'intérêt de la société». Malgré leurs tentatives de convaincre les victimes d'accepter l'intervention pénale, celles-ci refusent souvent de se présenter à la Cour ou, si elles s'y présentent, elles refusent de relater les événements tels qu'ils se sont réellement produits et passent sous silence les circonstances «aggravantes» nécessaires pour déterminer la gravité pénale de l'acte.

Pour une meilleure compréhension de cette attitude et de l'impact qu'elle peut avoir sur la qualification des actes, présentons quelques données sur les caractéristiques socio-économiques et relationnelles des parties qui ont vu leur conflit traité à la Cour municipale comme des voies de fait ainsi que sur les événements à l'origine d'une telle accusation. Plus de la moitié (54\%) des cas de voies de fait traités relèvent de la violence entre conjoints $(44 \%)$ ou ex-conjoints $(10 \%)$. De plus, 25 pour cent des cas concernent des relations familiales ou amicales. De nombreuses victimes de ces conflits interpersonnels vivaient avec l'auteur de l'incident violent lors des événements (40\%). Une autre particularité du traitement de ces cas est révélée par le portrait socioéconomique et juridique de cette clientèle.

Rappelons d'abord certaines données concernant les accusés:

- sexe de l'accusé: masculin

$95 \%$

— âge de l'accusé: entre 24 et 41 ans

$63 \%$

11. D. S. Elliott (1989), p. $459-460$. 
- accusés n'étant pas de race blanche

- pays d'origine de l'accusé autre que le Canada

- occupation de l'accusé

sans emploi $\quad 32 \%$

prestations sociales $23 \%$

- consommation de drogue ou d'alcool

lors des événements $32 \%$

- antécédents judiciaires ${ }^{12} \quad 45 \%$

— antécédents carcéraux $\quad 5 \%$

- accusés sous le coup d'une cause pendante $\quad 10 \%$

- accusés sous le coup d'une décision $\quad 11,5 \%$

Quant aux victimes, soulignons d'abord la pauvreté des informations notées dans les dossiers policiers et judiciaires. Les informations dont nous disposons pourraient être regroupées comme suit:

- sexe de la victime: féminin

- âge de la victime: entre 20 et 39 ans

- occupation de la victime:

sans emploi

prestations sociales

- occupation de la victime de violence conjugale:

sans emploi

prestations sociales

$\mathrm{Si}$, de façon générale, les données socio-économiques ne démontrent pas une vulnérabilité financière plus importante chez les victimes que chez les accusés, les victimes aux prises avec la violence conjugale sont beaucoup plus désavantagées. Il est possible que cette vulnérabilité financière rende difficile d'accepter la procédure de rupture que privilégient souvent les interventions. Il se peut que les auteurs qui se sont penchés sur cette question (voir par exemple Davis, 1988; Elliott, 1989) aient raison lorsqu'ils affirment que les sanctions (arrestation, détention, emprisonnement, amende, etc.) imposées

12. Il est nécessaire de noter que la variable «antécédents judiciaires» doit être considérée avec prudence. Les dossiers judiciaires dans lesquels nous avons réussi à retrouver le contenu de ces antécédents témoignent en effet de différences importantes quant aux infractions antérieures et aux sentences infligées. Pour certains accusés, il s'agit d'une infraction au code de la route; pour d'autres, d'accusations de toutes sortes (vols, voies de fait, etc.). Néanmoins, les rapports policiers donnent des informations sur les cas avec antécédents carcéraux. La proportion de ces cas est toutefois minime, soit 5 pour cent (ou 29 cas). À partir de cette information, nous pouvons croire que même si les antécédents judiciaires sont nombreux, ils consistent rarement en de graves infractions. 
à l'accusé peuvent causer autant de tort à la victime désireuse de poursuivre la vie en commun qu'à son agresseur.

Voici enfin quelques données sur les événements eux-mêmes:

- lieu du délit:

demeure commune $\quad 41 \%$

espace public $29 \%$

- type de blessure:

aucune trace $44 \%$

rougeurs, ecchymoses $44 \%$

coupures, fractures $12 \%$

— présence d'un rapport médical dans le dossier $\quad 7 \%$

Les événements se produisent dans une grande proportion dans la demeure commune de la victime et de l'agresseur. Notons à cet égard que pour être traités pénalement, il est indispensable que les incidents soient signalés ou constatés par les policiers, alors que ces lieux sont susceptibles d'engendrer des événements moins visibles que s'ils se produisent dans des lieux publics.

En ce qui concerne la "gravité» des actes, nous avons souligné que les événements traités devant la Cour municipale sont relativement moins «graves». Il est très rare que ces gestes violents causent des blessures graves, bien qu'il soit difficile, surtout dans les cas de conflits intrafamiliaux, de parler de la gravité des gestes sans rien dire de la manière dont ils sont posés ou vécus selon le contexte familial dans lequel ils s'inscrivent. Ces gestes définis comme des voies de fait risquent de n'être qu'un élément dans un processus qui ne débute ni ne se termine avec l'ouverture du dossier policier.

\section{LES FACTEURS CONSIDÉRÉS LORS DE LA QUALIFICATION DES ACTES VIOLENTS}

\section{LA QUALIFICATION DES ACTES DANS LES DEMANDES D'INTENTER DES PROCÉDURES}

\subsection{LES CHEFS D'ACCUSATION}

Nous venons de voir que les données sur la population dont le conflit a été traité comme constituant des voies de fait à la Cour municipale confirment le constat des auteurs selon lequel ce sont des groupes socialement défavorisés et juridiquement marqués qu'on retrouve surtout devant les cours criminelles. Notre objectif étant l'analyse des impacts éventuels de ces caractéristiques sur l'interprétation pénale des événements violents, examinons maintenant 
les décisions des policiers et des procureurs lorsqu'ils qualifient la gravité des actes en rapport avec les différentes variables légales et extra-légales susceptibles d'influencer cette activité. Ces décisions se prennent à trois stades des procédures: lors des demandes d'intenter des procédures, lors de la dénonciation et lors de la déclaration de la poursuite. C'est à travers ces trois phases que l'on examinera les accusations portées sur les mêmes événements. Dans 79 pour cent des cas (494 sur 625), la police a inscrit un seul chef d'accusation dans les demandes d'intenter des procédures, alors que dans 21 pour cent des cas (131), il y avait deux ou plusieurs chefs d'accusation, ces derniers ne comptant que 28 cas. La majorité des cas où les infractions de voies de fait étaient accompagnées d'une ou de plusieurs autres accusations, ce sont les voies de fait qui étaient inscrites comme premier chef, même si elles étaient qualifiées d'infractions sommaires, tandis que la seconde infraction était décrite comme un acte criminel (telles les menaces de mort). Les premiers chefs d'accusation sont représentés au tableau 1. Dans une proportion de 54 pour cent ( 337 cas), la police a simplement inscrit le chef d'accusation «voies de fait» sans se prononcer sur la qualification précise de l'infraction, ce qui est compréhensible vu l'ambiguité des dispositions relatives à la qualification des actes. Ce sont plutôt les cas où les policiers ont opté pour une accusation plus précise qui sont intéressants. En effet, dans 70 cas $(11 \%)$, la police a qualifié les voies de fait d'actes criminels (art. 266 a), et dans 171 cas (27\%), d'infractions punissables sur déclaration sommaire de culpabilité (art. $266 \mathrm{~b}$ ). Les accusations pour lésions corporelles (art. 267.1 (1) b) sont rares, soit 3,5 pour cent ( 22 cas). Si ces causes ne sont pas toujours renvoyées à la chambre criminelle de la Cour du Québec, conformément à ce que prescrivent les normes administratives émises par les directions de service de police, c'est peut-être en raison de l'ambiguïté qui entoure la définition des lésions corporelles, comme nous l'avons souligné plus haut. Quant aux autres infractions, tels l'agression sexuelle, l'agression armée, les menaces, le méfait, le fait de troubler la paix publique et les appels téléphoniques harassants, elles ne constituent que 4 pour cent des premiers chefs d'accusation.

Nous avons obtenu 124 informations sur les deuxièmes chefs d'accusation. Ce sont, en grande majorité, des méfaits ( $25 \%$, soit 31 cas), des menaces ( $24 \%$, soit 30 cas) et autres voies de fait (38\%, soit 47 cas). Les gestes violents envers la conjointe ou envers ses objets, les menaces à la victime pour la dissuader d'appeler la police se produisent généralement en même temps, et ce n'est qu'au plan juridique qu'ils seront traités séparément; d'abord par la police dans les différents chefs d'accusation, puis par les tribunaux dans les différents dossiers. Les voies de fait inscrites dans le deuxième chef signalent généralement la présence d'une deuxième victime dans l'affaire ou une agression contre un agent lors de l'intervention policière. 
Tableau 1

La distribution de fréquences de la qualification des actes par la police: premier chef d'accusation

\begin{tabular}{lrc}
\hline $\begin{array}{l}\text { Qualification des } \\
\text { actes par la police }\end{array}$ & Nombre & Pourcentage \\
\hline infliction de lésions & 22 & 3,5 \\
agression sexuelle & 2 & 0,3 \\
agression armée & 7 & 1,1 \\
voies de fait & 70 & 11,2 \\
acte criminel & 2 & 0,3 \\
menaces de mort & 171 & 27,4 \\
voies de fait & & \\
infraction sommaire & 337 & 53,9 \\
voies de faits & 1 & 0,1 \\
non spécifiées & 4 & 0,6 \\
intimidation & 1 & 0,1 \\
méfait & 2 & 0,3 \\
appels téléphoniques & 6 & 1,0 \\
harassants & & \\
dénonciation en vertu & $625(\mathrm{~N})$ & $100,0(\%)$ \\
de l'art. 745 & & \\
autres accusations & & \\
\hline Total & &
\end{tabular}

Valeurs présentes: $100 \%$

Comme il était impossible de traiter simultanément les données sur les deux chefs d'accusation ayant une variation dépassant la valeur 40 , il devenait nécessaire de faire un choix conceptuel et de regrouper ces informations pour les mettre en rapport avec d'autres variables. Nous avons donc groupé les données en trois catégories. Dans la première catégorie, nous retrouvons les cas où au moins un événement a été qualifié d'acte criminel; la deuxième catégorie regroupe les cas où l'on retrouve un ou plusieurs chefs d'accusation, mais tous constituant des infractions punissables par voie de déclaration sommaire de culpabilité; enfin, la troisième catégorie réunit les cas où un ou plusieurs chefs sont au dossier, mais sans suggestion concrète quant à la gravité des actes ou au type de procès. Cette reconstruction repose sur l'hypothèse selon laquelle la présence d'une infraction qualifiée d'acte criminel peut avoir un impact sur les autres décisions policières et judiciaires. Les distinctions légales relatives aux différentes procédures qui concernent l'acte criminel et l'infraction sommaire nous ont amenée à accepter cette reconstruction, mais nous sommes consciente que comme toute catégorisation, elle n'est pas neutre. 


\subsection{LA QUALIFICATION DES ACTES PAR LA POLICE ET LES VARIABLES LÉGALES}

Rappelons que pour déterminer la gravité «objective» des voies de fait, la nature de la blessure causée devrait, selon les dispositions du Code criminel, constituer le facteur principal. Les résultats de l'analyse, rapportés dans le tableau 2, laissent entrevoir que ce n'est pas toujours le cas en pratique. Nous pouvons constater notamment qu'il n'existe pas de différence significative entre les cas où les gestes violents ont pu causer des rougeurs ou des ecchymoses et ceux qui n'ont causé aucune trace de blessure. Si les gestes violents ayant causé des fractures ou des coupures (blessures dont on peut supposer qu'elles ne sont pas de «nature passagère») sont décrits comme des voies de fait constituant des actes criminels ( $36 \%$ par rapport à une moyenne de $18 \%$ ), il n'en reste pas moins que cette pratique de considérer l'acte le plus grave en cas de blessures graves n'est pas générale. En fait, 64 pour cent des cas (37 observations) ayant causé des fractures ou des coupures n'ont pas été qualifiés d'actes criminels.

D'autre part, les accusés ayant des antécédents judiciaires, des causes pendantes ou des décisions antérieures, telle la probation encore en vigueur, se voient plus souvent accusés d'actes criminels. La proportion de ces cas s'élève à 24 pour cent lorsqu'il y a des antécédents, à 30 pour cent dans les causes pendantes et à 34 pour cent si les accusés ont des sentences encore en vigueur, alors que la moyenne observée dans ces mises en relation ne s'élève qu'à 18 pour cent. Il se peut que les personnes ayant déjà de telles charges commettent des actes plus graves, mais il est également possible que les agents soient plus sévères lors de la qualification des actes ayant été commis par un suspect avec un dossier. Une fois de plus, la pratique ne semble pas uniforme puisque, dans 42 pour cent ( 92 cas) des cas d'actes qualifiés d'infractions sommaires, les policiers ont signalé la présence d'antécédents judiciaires.

\subsection{LA QUALIFICATION DES ACTES PAR LA POLICE ET LES VARIABLES EXTRA-LÉGALES}

Quant à la relation entre les parties et la qualification des actes, nous pouvons constater, tout d'abord, que les événements se produisant entre les personnes sans lien tendent à être renvoyés à la cour comme infractions sommaires. La proportion de cette qualification s'élève à 45,5 pour cent $(56$ cas) dans cette catégorie de lien, tandis qu'elle n'est que de 39 pour cent pour l'échantillon. Au sujet des conjoints, par contre, on peut observer une surreprésentation de voies de fait non qualifiées, leur proportion s'elevant à 50 pour cent ( $130 \mathrm{cas}$ ), comparativement à la moyenne de 43 pour cent. La tendance à la non-qualification de ces actes s'explique peut-être par la précarité 
et la vulnérabilité des preuves dans ces cas et par le fait que les policiers savent que ces victimes, souvent, retirent leur plainte. Cela peut avoir un impact sur la décision, ou plutôt sur la «non-décision» des agents policiers, qui laissent volontiers cette tâche aux procureurs.

Dans la catégorie ex-conjoint, c'est la tendance inverse qui apparaît. Ici, les qualifications «concrètes» l'emportent: 53 pour cent ( 32 cas) des événements reprochés à ces personnes ont été qualifiés d'infractions sommaires, comparativement à une moyenne de 39 pour cent. La proportion des cas qualifiés d'actes criminels est aussi plus élevée, avec 23 pour cent ( 14 cas), dans la catégorie des ex-conjoints que dans les autres. Par contre, nous observons une proportion beaucoup moins importante de voies de fait non spécifiées, soit 23 pour cent ( 14 cas), comparativement à la moyenne de 43 pour cent et à 50 pour cent pour les conjoints. Nous estimons possible que ces décisions "concrètes» concernant l'accusation des ex-conjoints violents soient liées à la certitude qu'ont les policiers que la victime persévérera à poursuivre son «agresseur» devant les tribunaux.

Les résultats concernant le lieu de l'incident indiquent à leur tour que les policiers hésitent moins souvent à qualifier les actes d'une façon précise dans les cas des événements qui se produisent dans des lieux publics et qu'ils reconnaissent aussi plus fréquemment les voies de fait comme des actes criminels. La proportion des cas non-spécifiés n'est que de 35,5 pour cent $(52$ cas) dans la catégorie des lieux publics, alors que la moyenne est de 43 pour cent. De même, la proportion des actes criminels s'élève à 24 pour cent (38 cas) pour ces lieux, alors qu'elle n'est que de 18 pour cent dans l'échantillon. Il nous semble que le nombre plus élevé de qualifications «sûres» et plus graves peut se rapporter à la visibilité accrue de ces événements et au contexte interpersonnel moins intime des parties, donc plus clair pour les agents.

\section{LA QUALIFICATION DE L'ACTE LORS DE LA DÉNONCIATION}

Lorsqu'une demande d'intenter des procédures est soumise à la Cour municipale, les greffiers trient les différentes accusations portées et les enregistrent dans différents dossiers judiciaires, selon qu'il s'agit de voies de fait, de menaces, de méfaits ou d'autres infractions. C'est lors de la comparution de l'accusé que ces dossiers seront regroupés. Si l'accusé est libéré ou acquitté dans le cas d'un dossier, la poursuite peut continuer dans l'autre.

Il serait intéressant de voir dans quelle mesure le découpage des événements connexes dans différents dossiers est lié aux décisions prises. Nous n'avions pas pour objectif l'étude de cette question, bien qu'elle mériterait, à notre avis, un examen plus poussé. Nous avons néanmoins fusionné 
Tableau 2

Qualification des événements par la police

\begin{tabular}{|c|c|c|c|c|}
\hline \multirow{2}{*}{$\begin{array}{c}\text { Variables reliees } \\
\text { a la } \\
\text { qualification } 13\end{array}$} & \multirow[b]{2}{*}{$\begin{array}{c}\text { Acte } \\
\text { criminel }\end{array}$} & \multicolumn{2}{|c|}{ Qualification de l'acte } & \multirow[b]{2}{*}{ Khi carré } \\
\hline & & $\begin{array}{l}\text { Infraction } \\
\text { sommaire }\end{array}$ & $\begin{array}{c}\text { Sans } \\
\text { précision }\end{array}$ & \\
\hline $\begin{array}{l}\text { Blessure } \\
\text { causée }\end{array}$ & 18 & 39 & 43 & $\begin{array}{c}17,5 \mathrm{dl}=4 \\
(p \leqslant 0,0015)\end{array}$ \\
\hline $\begin{array}{l}\text { aucune } \\
\text { trace }\end{array}$ & $\begin{array}{l}12,8 \\
(28)\end{array}$ & $\begin{array}{l}40,4 \\
(88)\end{array}$ & $\begin{array}{l}46,8 \\
(102)\end{array}$ & \\
\hline $\begin{array}{l}\text { rougeurs ou } \\
\text { ecchymoses }\end{array}$ & $\begin{array}{l}17,8 \\
(39)\end{array}$ & $\begin{array}{l}40,6 \\
(89)\end{array}$ & $\begin{array}{l}41,6 \\
(91)\end{array}$ & \\
\hline $\begin{array}{l}\text { fracture ou } \\
\text { coupure }\end{array}$ & $\begin{array}{l}36,2 \\
(21)\end{array}$ & $\begin{array}{l}29,3 \\
(17)\end{array}$ & $\begin{array}{l}34,5 \\
(20)\end{array}$ & \\
\hline $\begin{array}{l}\text { Antécédents } \\
\text { judiciaires }\end{array}$ & 18 & 39 & 43 & $\begin{array}{c}9,0 \mathrm{dl}=2 \\
(p \leqslant 0,0108)\end{array}$ \\
\hline oui & $\begin{array}{l}23,6 \\
(59)\end{array}$ & $\begin{array}{l}36,8 \\
(92)\end{array}$ & $\begin{array}{l}39,6 \\
(99)\end{array}$ & \\
\hline non & $\begin{array}{l}13,7 \\
(42)\end{array}$ & $\begin{array}{l}40,8 \\
(125)\end{array}$ & $\begin{array}{l}45,4 \\
(139)\end{array}$ & \\
\hline $\begin{array}{l}\text { Causes } \\
\text { pendantes }\end{array}$ & 18 & 39 & 43 & $\begin{array}{c}5,9 \mathrm{dl}=2 \\
(\mathrm{p} \leqslant 0,0528)\end{array}$ \\
\hline oui & $\begin{array}{l}30,2 \\
(16)\end{array}$ & $\begin{array}{l}35,8 \\
(19)\end{array}$ & $\begin{array}{l}34,0 \\
(18)\end{array}$ & \\
\hline non & $\begin{array}{l}16,9 \\
(85)\end{array}$ & $\begin{array}{l}39,4 \\
(198)\end{array}$ & $\begin{array}{l}43,7 \\
(220)\end{array}$ & \\
\hline $\begin{array}{l}\text { Décisions } \\
\text { judiciaires }\end{array}$ & 18 & 39 & 43 & $\begin{array}{c}12,0 \mathrm{dl}=2 \\
(\mathrm{p} \leqslant 0,0016)\end{array}$ \\
\hline oui & $\begin{array}{l}34,4 \\
(22)\end{array}$ & $\begin{array}{l}29,7 \\
(19)\end{array}$ & $\begin{array}{l}35,9 \\
(23)\end{array}$ & \\
\hline non & $\begin{array}{l}16,1 \\
(79)\end{array}$ & $\begin{array}{l}40,2 \\
(198)\end{array}$ & $\begin{array}{r}43,7 \\
(220)\end{array}$ & \\
\hline $\begin{array}{l}\text { Lien } \\
\text { entre les parties }\end{array}$ & 18 & 39 & 43 & $\begin{array}{r}18,3 \mathrm{dl}=6 \\
(p \leqslant 0,0055)\end{array}$ \\
\hline conjoint & $\begin{array}{l}17,0 \\
(22)\end{array}$ & $\begin{array}{l}32,8 \\
(85)\end{array}$ & $\begin{array}{l}50,2 \\
(130)\end{array}$ & \\
\hline ex-conjoint & $\begin{array}{l}23,3 \\
(14)\end{array}$ & $\begin{array}{l}53,3 \\
(32)\end{array}$ & $\begin{array}{l}23,3 \\
(14)\end{array}$ & \\
\hline sans lien & $\begin{array}{l}17,9 \\
(22)\end{array}$ & $\begin{array}{l}45,5 \\
(56)\end{array}$ & $\begin{array}{l}36,6 \\
(45)\end{array}$ & \\
\hline $\begin{array}{l}\text { Lieu } \\
\text { du délit }\end{array}$ & 18 & 39 & 43 & $\begin{array}{c}16,5 \mathrm{dl}=8 \\
(p \leqslant 0,0357)\end{array}$ \\
\hline $\begin{array}{l}\text { demeure } \\
\text { commune }\end{array}$ & $\begin{array}{l}17,1 \\
(38)\end{array}$ & $\begin{array}{l}34,2 \\
(76)\end{array}$ & $\begin{array}{l}48,6 \\
(108)\end{array}$ & \\
\hline lieu public & $\begin{array}{l}23,8 \\
(38)\end{array}$ & $\begin{array}{l}43,8 \\
(70)\end{array}$ & $\begin{array}{l}32,5 \\
(52)\end{array}$ & \\
\hline
\end{tabular}

13. Notons concernant ces variables que nous n'avons pas rapporté toutes les catégories mais uniquement les plus importantes. 
les informations obtenues à partir des différents dossiers judiciaires concernant les mêmes accusés, afin d'éviter d'analyser des décisions judiciaires dont on ne connaîtrait pas au moins les composantes les plus importantes. Par conséquent, les variables décrivant la qualification de l'acte lors de la dénonciation et de la déclaration de la poursuite englobent les différents chefs d'accusation dans un dossier ainsi que les accusations portées contre le même accusé dans d'autres dossiers, et ceci en rapport avec les mêmes événements.

Quant aux changements survenus dans la qualification des actes lors de la dénonciation par rapport aux demandes d'intenter des procédures, nous pouvons observer deux tendances. D'une part, lors de la dénonciation, la gravité des événements de même que le nombre d'accusations tendent à être réduits. D'autre part, et c'est ce qui arrive le plus fréquemment, les événements tendent à être requalifiés à un niveau plus ambigu, avec seulement une référence à l'article 265 du Code criminel, sans définition précise quant à la gravité de l'acte. Nous ignorons dans quelle mesure les consultations entre les agents de liaison et les procureurs peuvent expliquer ces modifications, mais la tendance vers la non-qualification fait ressortir le pouvoir discrétionnaire des procureurs, ceux-ci décidant du trajet des causes avant même qu'elles n'arrivent au stade judiciaire.

\section{LA QUALIFICATION DES ACTES LORS DE LA DÉCLARATION DE LA POURSUITE}

\subsection{LE LIEN ENTRE LA QUALIFICATION DES ACTES PAR LA POLICE ET CELLE PAR LA POURSUITE}

Nous avons mis en relation les actes d'accusation lors de la demande d'intenter des procédures et lors de la déclaration de la poursuite afin d'analyser la nature des changements survenus. Deux phénomènes émergent. Il semble d'abord que les «charges» contre l'accusé diminuent souvent à cette phase: les accusations portées pour voies de fait constituant des actes criminels se transformant dans bien des cas en accusations pour infractions punissables par voie de déclaration sommaire de culpabilité, ou encore c'est le nombre de chefs d'accusation dans les dossiers judiciaires qui diminue par rapport à celui défini par les policiers. Nous observons enfin que les procureurs ont tendance à abandonner les poursuites pour les différentes accusations sommaires et à ne poursuivre l'accusé que pour une infraction de voies de fait décrite comme un acte criminel.

Dans les paragraphes qui suivent, nous présenterons le résultat de ces changements en rapport avec les variables légales et extra-légales. Notons que les infractions qui sont décrites par la poursuite comme des voies de fait 
causant des lésions corporelles ou des voies de fait graves ont été renvoyées à la Cour des sessions de la paix (aujourd'hui Cour du Québec, chambre criminelle). Notre analyse ne concerne donc que les cas qui sont restés à la Cour municipale et où le procureur avait à choisir de procéder pour acte criminel ou pour infraction punissable sur déclaration sommaire de culpabilité $(\mathbf{N}=596$ cas $)$.

\subsection{LES RAPPORTS ENTRE LA QUALIFICATION DES ACTES PAR LA POURSUTTE ET LES VARIABLES LÉGALES}

Contrairement à ce qu'on pouvait observer dans le cas des accusations suggérées par la police, les accusations au moment de la poursuite ne sont pas significativement liées à la nature des blessures causées. En effet, dans la catégorie des blessures causant des fractures ou coupures, nous observons une proportion légèrement plus élevée d'actes criminels, soit 18 pour cent ( 9 cas), alors que la moyenne n'est que de 12 pour cent. Dans les autres catégories de blessures, nous n'observons pas de différences. Par contre, tout comme au stade de l'enquête policière, le passé judiciaire de l'accusé pèse contre lui lorsque la poursuite détermine la gravité de son acte (tableau 3). En effet, dans la catégorie des personnes avec antécédents judiciaires, la proportion d'actes criminels s'élève à 19 pour cent ( 44 cas), par rapport à 9 pour cent ( $27 \mathrm{cas})$ chez les accusés sans antécédents. Elle s'élève à 28 pour cent dans le cas des personnes ayant des causes pendantes, à 26 pour cent dans le cas des accusés avec une autre décision encore en vigueur à leur charge, alors que la moyenne n'est que de 12 pour cent pour les deux variables.

\subsection{LES RAPPORTS ENTRE LA QUALIFICATION DE L'ACTE PAR LA POURSUITE ET LES VARIABLES EXTRA-LÉGALES}

Parmi les variables extra-légales, seul le lien entre les parties s'est révélé significativement relié à la qualification des actes par la poursuite. Tout comme c'était le cas à la police, la gravité des actes qualifiée par les procureurs differe significativement en fonction des différentes catégories de lien. Les personnes sans aucun lien sont plus susceptibles d'être accusées d'infraction sommaire, soit dans une proportion de 80 pour cent ( $96 \mathrm{cas}$ ), par rapport à une moyenne de 67 pour cent pour l'ensemble de l'échantillon et à une proportion de 58 pour cent (143 cas) dans le cas des conjoints. Par contre, les conjoints sont plutôt surreprésentés par rapport à la moyenne pour voies de fait non qualifiées (28\%, alors que la moyenne est de $19 \%$ ), ce qui reflète les cas où la plainte a été retirée ou la procédure arrêtée par le procureur. Concernant les ex-conjoints, nous constatons que la gravité de la qualification des actes augmente; la proportion d'actes criminels est de 15,5 pour cent ( 9 cas), la moyenne n'étant que de 13 pour cent. Les actes demeurant non qualifiés dimi- 
nuent à leur tour à 12 pour cent $(7 \mathrm{cas})$, cette même proportion étant de 28 pour cent pour les conjoints.

Tableau 3

Qualification des événements par la poursuite

\begin{tabular}{|c|c|c|c|c|}
\hline $\begin{array}{c}\text { Variables reliées } \\
\text { à la } \\
\text { qualification }\end{array}$ & $\begin{array}{c}\text { Acte } \\
\text { criminel }\end{array}$ & $\begin{array}{l}\quad \text { Quali } \\
\text { Infraction } \\
\text { sommaire }\end{array}$ & $\begin{array}{l}\text { ion de l'acte } \\
\text { Sans } \\
\text { précision }\end{array}$ & Khi carré \\
\hline $\begin{array}{l}\text { Blessure } \\
\text { causée }\end{array}$ & 12 & 70 & 18 & non significatif \\
\hline $\begin{array}{l}\text { aucune } \\
\text { trace }\end{array}$ & $\begin{array}{l}10,6 \% \\
(23)\end{array}$ & $\begin{array}{c}74,5 \% \\
(161)\end{array}$ & $\begin{array}{c}14,8 \% \\
(32)\end{array}$ & \\
\hline $\begin{array}{l}\text { rougeurs ou } \\
\text { ecchymoses }\end{array}$ & $\begin{array}{l}12,2 \% \\
(26)\end{array}$ & $\begin{array}{l}68,1 \% \\
(145)\end{array}$ & $\begin{array}{l}19,7 \% \\
(42)\end{array}$ & \\
\hline $\begin{array}{l}\text { fractures ou } \\
\text { coupures }\end{array}$ & $\begin{array}{l}18,4 \% \\
\text { (9) }\end{array}$ & $\begin{array}{c}61,2 \% \\
(30)\end{array}$ & $\begin{array}{c}20,4 \% \\
(10)\end{array}$ & \\
\hline $\begin{array}{l}\text { Antécédents } \\
\text { judiciaires }\end{array}$ & 13 & 70 & 17 & $\begin{array}{c}10,6 \mathrm{dl}=2 \\
(p \leqslant 0,0049)\end{array}$ \\
\hline oui & $\begin{array}{c}18,6 \% \\
(44)\end{array}$ & $\begin{array}{c}66,1 \% \\
(156)\end{array}$ & $\begin{array}{c}15,3 \% \\
(36)\end{array}$ & \\
\hline non & $\begin{array}{l}9,2 \% \\
(27)\end{array}$ & $\begin{array}{l}71,5 \% \\
(211)\end{array}$ & $\begin{array}{c}19,3 \% \\
(57)\end{array}$ & \\
\hline $\begin{array}{l}\text { Causes } \\
\text { pendantes }\end{array}$ & 13 & 70 & 17 & $\begin{array}{c}10,5 \mathrm{dl}=2 \\
(p \leqslant 0,0054)\end{array}$ \\
\hline oui & $\begin{array}{c}28,0 \% \\
\text { (14) }\end{array}$ & $\begin{array}{c}60,0 \% \\
(30)\end{array}$ & $\begin{array}{c}12,0 \% \\
(6)\end{array}$ & \\
\hline non & $\begin{array}{c}11,9 \% \\
(57)\end{array}$ & $\begin{array}{l}70,1 \% \\
(337)\end{array}$ & $\begin{array}{c}18,2 \% \\
(87)\end{array}$ & \\
\hline $\begin{array}{l}\text { Décisions } \\
\text { judiciaires }\end{array}$ & 13 & 70 & 17 & $\begin{array}{c}9,3 d\}=2 \\
(p \leqslant 0,0097)\end{array}$ \\
\hline oui & $\begin{array}{c}26,3 \% \\
(15)\end{array}$ & $\begin{array}{c}59,6 \% \\
(34)\end{array}$ & $\begin{array}{c}14,0 \% \\
(8)\end{array}$ & \\
\hline non & $\begin{array}{c}11,8 \% \\
(56)\end{array}$ & $\begin{array}{c}70,3 \% \\
(333)\end{array}$ & $\begin{array}{c}17,9 \% \\
(85)\end{array}$ & \\
\hline $\begin{array}{l}\text { Lien } \\
\text { entre les parties }\end{array}$ & 13 & 67 & 19 & $\begin{array}{c}28,4 \mathrm{dl}=6 \\
(p \leqslant 0,0001)\end{array}$ \\
\hline conjoint & $\begin{array}{c}14,1 \% \\
(35)\end{array}$ & $\begin{array}{c}57,7 \% \\
(143)\end{array}$ & $\begin{array}{c}28,2 \% \\
(70)\end{array}$ & \\
\hline ex-conjoint & $\begin{array}{c}15,5 \% \\
(9)\end{array}$ & $\begin{array}{c}72,4 \% \\
(42)\end{array}$ & $\begin{array}{c}12,1 \% \\
(7)\end{array}$ & \\
\hline sans lien & $\begin{array}{c}12,5 \% \\
(15)\end{array}$ & $\begin{array}{c}80,0 \% \\
(96)\end{array}$ & $\begin{array}{l}7,5 \% \\
(9)\end{array}$ & \\
\hline
\end{tabular}

\section{EN GUISE DE CONCLUSION}

Nous avons vu l'importance que revêt l'interprétation de la gravité des actes violents lorsque ceux-ci se transforment en infractions de voies de fait 
et quand leurs auteurs font l'objet des différentes mesures pénales. Ces gestes violents sont tout d'abord évalués par les victimes elles-mêmes, leur décision concernant la demande d'intervention pour solutionner le conflit semblant dépendre du contexte social, qui lui-même donne un sens, une signification à la gravité de l'acte. Lorsque les causes sont acheminées dans le système pénal, les gestes et les acteurs sociaux font l'objet de nouvelles évaluations qui qualifieront les actes conformément à l'interprétation juridique qu'on donnera de leur gravité.

Étant donné l'importance que l'on accorde au principe de proportionnalité de la peine à la gravité de l'acte et au degré de responsabilité de son auteur, nous avons interrogé nos données à ce sujet. Nous voulions surtout savoir dans quelle mesure, à l'intérieur d'un groupe relativement homogène d'infractions comme celui des voies de fait, il était possible ou souhaitable de qualifier des actes semblables au point de vue juridico-pénal d'une façon semblable.

Pour mieux comprendre le fonctionnement de ce processus, nous avons traité les articles du Code criminel définissant les voies de fait. Le bref survol de ces dispositions légales souvent ambiguës nous a montré qu'il était difficile, même au plan juridique, de définir d'une façon uniforme ce qui doit être considéré comme des voies de fait «graves» ou «moins graves».

L'analyse des accusations portées par les agents des différents paliers indiquent qu'ils ne considèrent pas les mêmes événements de façon similaire et que la notion de "gravité» diffère de l'un à l'autre. D'une façon générale, les accusations suggérées par la police dans les demandes d'intenter des procédures tendent à être qualifiées plus sévèrement que lors de la dénonciation, où les accusations sont réduites à un niveau plus ambigu, pour être ensuite requalifiées moins gravement par la poursuite, la plupart du temps comme infractions sommaires. Si la gravité des actes varie d'un stade du processus à l'autre, il en va de même pour les variables significativement associées à la qualification des actes.

Nous avons également pu observer que certaines variables, tel le lien entre les parties, influent sur les décisions des agents, non seulement dans le choix de procéder par voie d'accusation ou par procédure sommaire, mais aussi dans bien des cas sur le degré de certitude des agents. Par ailleurs, il semble que le degré d'incertitude des accusations portées par les policiers soit directement proportionnel à celle des agents quant à la persévérance de la victime à poursuivre son agresseur.

À la lumière de ces résultats, il nous semble que l'application du principe de proportionnalité dans ces cas se bute à plus d'un problème. Déjà, les défi- 
nitions normatives ne facilitent pas l'établissement adéquat de ce rapport de proportionnalité. De plus, l'évaluation des éléments de gravité, préalable à l'établissement de ce rapport, semble être inévitablement affectée par des facteurs incompatibles avec ce principe. Le principe de proportionnalité met en relief entre autres les événements du passé comme base de considération. Or ce regard vers le passé est très difficile dans le contexte de conflits intrafamiliaux qui s'inscrivent dans un processus incluant les événements du passé, du présent et du futur. Nous estimons que l'évaluation «objective» de la gravité de l'acte risquerait de ne pas prendre suffisamment en considération les circonstances variables de ces incidents et ainsi d'aller à l'encontre de l'intérêt de la victime. Pour résoudre son conflit, selon le point de vue de la victime, il n'est peut-être pas souhaitable d'appliquer des sanctions rigoureusement proportionnelles à la gravité objective de l'acte et au degré de responsabilité de son auteur, comme le veulent les dispositions pénales.

Par ailleurs, de nombreuses victimes, comme les études victimologiques l'indiquent, passent sous silence des circonstances dont la révélation entraînerait une sentence qu'elles ne sont pas prêtes à assumer. Leur résistance à une coopération éventuelle avec la justice rend l'application du principe de proportionnalité plus difficile. Nous estimons que dans ces cas particuliers, le fait de favoriser la résolution des conflits plutôt que l'application de sanctions «justes et proportionnelles» à la gravité de l'acte et au degré de responsabilité de son auteur est inévitable. Reste à se demander si cette résolution des conflits devrait être résolue dans le cadre du système pénal.

\section{BIBLIOGRAPHIE}

BENZVY-MILLER, S. (1988), Une étude empirique des circonstances atténuantes et aggravantes dans les appels de sentences en Alberta et au Québec entre 1980 et 1985, rapport de recherche de la Commission canadienne sur la détermination de la peine, Ministère de la Justice, Canada.

BARIL, M. (1984), L'Envers du crime, Montréal, École de criminologie.

BLACK, D. (1989), Sociological Justice, New York et Oxford, Oxford University Press.

BRODEUR, J.-P. (1990), «Réforme et difformités pénales», Déviance et société, vol. XIV, $\mathrm{n}^{\circ} 2$, p. $133-156$.

BRODEUR, J.-P. (1990), «To Seem or not To Seem. A Brief Reply to Dr. Andrews», Canadian Journal of Criminology, vol. 32, $\mathrm{n}^{\circ} 3$, p. 525-528.

BRODEUR, J.-P. (1985), «Réforme pénale et sentences : Expériences nord-américaines», Déviance et société, vol. IX, $\mathrm{n}^{\circ} 3$, p. 165-200.

CHAPMAN, D. (1968), Sociology and the Stereotype of the Crime, Londres, Tavistock Publication. 
COMMISSION CANADIENNE SUR LA DÉTERMINATION DE LA PEINE (1987), Réformer la sentence: une approche canadienne, Ottawa, Ministère des Approvisionnements et Services Canada.

COMMISSION DE RÉFORME DU DROIT DU CANADA (1984), Les Voies de fait, document de travail $n^{\circ} 3$, Ottawa, Ministère des Approvisionnements et Services Canada.

DAVIS, N. J. (1988), «Battered Women: Implication for Social Control», in Contemporary Crisis, vol. 12, p. 345-372.

DOOB, A. (1990), «Community Sanctions and Imprisonment: Hoping for a Miracle but not Bothering Even to Pray for it", Canadian Journal of Criminology, vol. $32, n^{\circ} 3$, p. 415-428.

DOOB, A., PARK, N. W. (1987), «Computerized Sentencing Process», Criminal Law Quarterly, vol. 30, p. 54-72.

DUMONT, H. (1983), Le Contrôle judiciaire de la criminalité familiale, Montréal, Thémis.

ELLIOTT, D. S. (1989), "Criminal Justice Procedures in Family Violence Crimes», in Michael Tonry (éd.), Crime and Justice. A Review of Research, vol. 2.

ERICSON, R. (1981), Making Crime: A Study of Police Detective Work.

ERICSON, R., BARANEK, P. (1982), The Ordering of Justice: A Study of Accused Persons as Dependents in the Criminal Process, Toronto, University of Toronto Press.

FEELEY, M. M., (1979), The Process is the Punishment. Handling Cases in a Lower Criminal Court, New York, Russell Sage Foundation.

GABOR, T. (1990), «Looking Back or Looking Forward: Retributivism and the Canadian Sentencing Commission's Proposals», Canadian Journal of Criminology, vol. 32, $\mathrm{n}^{\circ} 3$, p. 537-546.

GOFFMAN, E. (1968), Asiles, Paris, Les Éditions de Minuit.

HOGARTH, J. (1971), Sentencing as a Human Process, Toronto, University of Toronto Press.

LANDREVILLE, P. (1983), Normes sociales et normes pénales: Notes pour une analyse socio-politique des normes, École de criminologie, Université de Montréal.

PIRES, A. P. (1990), «Le devoir de punir: le rétributivisme face aux sanctions communautaires", Revue canadienne de criminologie, vol. 32, n³, p. 441-460.

PIRES, A. P. (1987), «La réforme pénale au Canada: l'apport de la Commission canadienne sur la détermination de la peine», Criminologie, vol. XX, $\mathrm{n}^{\circ} 2$, p. 11-57.

PIRES, A. P. et LANDREVILLE, P. (1985), «Les recherches sur les sentences et le culte de la loi", L'Année sociologique, p. 83-113.

POIRIER, R. (1988), La Résoiution des causes pénales par les avocats de la défense, rapport de recherche de la Commission canadienne sur la détermination de la peine, Ministère de la Justice, Canada.

ROBERT, Ph. et FAUGERON, C. (1981), Les Forces cachées de la justice, Paris, Édition Centurion.

ROBERTS, J. (1990), «Sentencing in Canada: The Context for Reform», Canadian Journal of Criminology, vol. $32, \mathrm{n}^{\circ}$ 3, p. 381-386.

RUBY, C. C. (1987), Sentencing, $3^{\mathrm{e}}$ édition, Toronto et Vancouver, Butterworths.

STATISTTQUES (1987-1988), Violence conjugale, Québec, Ministère de la Sécurité publique, Direction générale de la sécurité et de la prévention.

VON HIRSCH, A. (1990), «Why Have Proportionate Sentences? A Reply to Professor Gabor», Canadian Journal of Criminology, vol. 32, $\mathrm{n}^{\circ} 3$, p. 547-550. 
VON HIRSCH, A. (1990), "The Ethics of Community-based Sanctions», Crime and Delinquency, vol. $36, \mathrm{n}^{\circ} 1$, p. 162-173.

VON HIRSCH, A. (1985), Past or Future Crimes: Deservedness and Dangerousness in the Sentencing of Criminals, New Brunswick, N.J., Rutgers University Press. 